\title{
Importance of $R N F 213$ polymorphism on clinical features and long-term outcome in moyamoya disease
}

\author{
*Eun-Hee Kim, MD,' Mi-Sun Yum, MD, PhD, ${ }^{1}$ Young-Shin Ra, MD, PhD, ${ }^{3}$ Jun Bum Park, MD, PhD, ${ }^{3}$ \\ Jae Sung Ahn, MD, PhD, ${ }^{3}$ Gu-Hwan Kim, PhD, ${ }^{2}$ Hyun Woo Goo, MD, PhD, ${ }^{4}$ Tae-Sung Ko, MD, PhD, ${ }^{1}$ \\ and Han-Wook Yoo, MD, PhD ${ }^{1,2}$
}

${ }^{1}$ Department of Pediatrics; ${ }^{2}$ Medical Genetics Clinic and Laboratory, Asan Medical Center Children's Hospital; and Departments of ${ }^{3}$ Neurosurgery and ${ }^{4}$ Radiology, Asan Medical Center, University of Ulsan College of Medicine, Seoul, Republic of Korea

\begin{abstract}
OBJECTIVE Moyamoya disease (MMD) is an idiopathic cerebrovascular occlusive disorder prevalent in East Asia. In the pathogenesis of MMD, the important role of genetic factors is being elucidated, and RNF213 has recently been identified as a susceptibility gene for MMD. The aim of this retrospective study was to investigate the RNF213 genotype in patients with MMD and to determine their genotype-phenotype associations.

METHODS The study involved 165 Korean MMD patients from 155 unrelated families who were diagnosed with MMD at a single center from 1995 to 2013. Their demographic, radiological, and clinical findings were evaluated. Direct sequencing of the major RNF213 single nucleotide polymorphisms was performed. The association of the common RNF213 variant with MMD risk was evaluated using historical controls for comparison. Correlations between RNF213 genotype and phenotype were statistically analyzed.

RESULTS The c.14429G>A (p.R4810K) variant was identified in 125 (75.8\%) of 165 MMD patients. Most patients (112) were heterozygous, and 13 patients had 2 copies of the $c .14429 \mathrm{G}>\mathrm{A}$ variant. A novel heterozygous variant, $c .12086 \mathrm{~A}>\mathrm{G}$ (p.Q4029R), was found in 1 additional patient. The minor allele frequency of the c.14429G>A variant was significantly higher in the MMD group (138 [41.8\%] of 330 patients) than in the control group (8 [1.36\%] of 588 subjects; $p<0.001)$. The c.14429G>A (p.R4810K) variant significantly increased the risk of MMD in Korean patients, with an OR of 52.11 ( $p<$ 0.001 ) compared with controls. Moreover, c.14429G>A (p.R4810K) genotypes occurred more frequently in patients with a family history of MMD. The homozygous variant was highly associated with early-onset MMD (age at onset $<5$ years), cerebral infarction at diagnosis, and cognitive impairment in long-term outcome.
\end{abstract}

CONCLUSIONS The findings indicate that the c.14429G>A (p.R4810K) allele of RNF213 is strongly associated with Korean patients with MMD. The homozygous c.14429G>A (p.R4810K) variant is particularly related to early-onset MMD, severe symptomatic manifestations at diagnosis, and poor prognosis. This genotypic variant may be a useful biomarker for early-onset MMD or unstable MMD with cerebral infarction, which requires early diagnosis and revascularization treatment.

http://thejns.org/doi/abs/10.3171/2015.4.JNS142900

KEY WORDS moyamoya disease; RNF213; single nucleotide polymorphism; phenotype; vascular disorders

$\mathrm{F}$ Tound in East Asia, particularly Korea and Japan, moyamoya disease (MMD) is an uncommon cerebrovascular disease characterized by progressive occlusion of the terminal portions of the internal carotid arteries (ICAs). ${ }^{11,12}$ Recent epidemiological study has revealed that the number of patients with MMD in Korea is increasing and that the prevalence rate of MMD per 100,000 people increased to 9.0 in 2008 from 5.2 in $2004 .^{5}$ Moyamoya disease is a relatively common cause of child- hood stroke,,$^{1,19}$ and it can lead to irreversible neurological deficits and cognitive impairment; therefore, early diagnosis and proper therapeutic approaches are important.

The etiology of MMD remains unclear, but several epidemiological risk factors are apparent. These include East Asian ethnicity, female sex, and family history of MMD. Some known genetic disorders present with moyamoyalike findings on cerebral angiography, suggesting that genetic factors may underlie MMD pathogenesis. ${ }^{13,25} \mathrm{Al}-$

ABBREVIATIONS ICA = internal carotid artery; $M C A=$ middle cerebral artery; $\mathrm{MMD}=$ moyamoya disease; $\mathrm{PCA}=$ posterior cerebral artery; $\mathrm{SNP}=$ single nucleotide polymorphism; TIA = transient ischemic attack.

SUBMITTED December 22, 2014. ACCEPTED April 7, 2015.

INCLUDE WHEN CITING Published online October 2, 2015; DOI: 10.3171/2015.4.JNS142900.

* Drs. E. H. Kim and Yum contributed equally to this work. 
though authors of recent genetic studies have made efforts to identify several candidate loci (chromosomes 3p24-26, $6 \mathrm{q} 25,8 \mathrm{q} 23,12 \mathrm{p} 12$, and $\left.17 \mathrm{q} 25^{4,6,24,31}\right)$ and several susceptibility genes (ACTA2, RPTOR, PDGFRB, MMP3, and $T G F B 1^{2,3,15,16}$ ) related to MMD, the RING (Really Interesting New Gene) finger protein 213 gene (RNF213) is the only susceptibility gene for MMD identified by both genome-wide association studies and exome sequencing.

RNF213 at locus $17 \mathrm{q} 25.3$ is $135.42 \mathrm{~kb}$ in size, has 69 exons, and encodes a zinc finger protein involved in protein-protein interactions. ${ }^{7,17,18,21,28}$ Several single nucleotide polymorphisms (SNPs) in RNF213, including c.14576G $>\mathrm{A}$ (p.R4859K) and c.14429G $>$ A (p.R4810K), were identified as variants with a strong susceptibility for MMD. ${ }^{7,18,20,21}$ The risk of MMD was significantly increased with the c.14576G $>$ A (p.R4859K) variant, with an odds ratio (OR) of $190.8,7$ and homozygotes exhibited severe and diverse vasculopathy phenotypes in a Japanese study. ${ }^{21}$ The c.14429G $>$ A (p.R4810K) variant was suggested to have the ischemic-type MMD in the Chinese Han population. ${ }^{28}$ Although MMD is especially prevalent in East Asia, including Korea, previous research on these RNF213 variants and their clinical implications have largely been conducted in Japan and China. This is the first study of $R N F 213$ polymorphism associated with clinical features and long-term outcomes in Korean patients with MMD. Our aim in the present study was to investigate the polymorphism variants of RNF213 in Korean patients with MMD and to determine whether this genotype has significant phenotypic correlation.

\section{Methods \\ Study Subjects}

The study involved 165 Korean patients ( 64 males, 101 females, mean age $21.3 \pm 13.6$ years, range $2.4-70.5$ years) from 155 unrelated families who had been diagnosed with MMD at the Asan Medical Center in Seoul, Korea, in the period from 1995 to 2013. The medical records of these patients were thoroughly reviewed to confirm the diagnosis of MMD, which had been based on conventional angiography (150 patients) or MR angiography (15 patients) findings interpreted by 1 radiologist. The diagnosis of MMD requires all of the following findings: 1) stenosis or occlusion of the terminal portion of the intracranial ICA or the proximal portions of the anterior cerebral artery (ACA) and/or middle cerebral artery (MCA); 2) abnormal vascular networks in the vicinity of the occlusive or stenotic lesions in the arterial phase; 3) bilateral findings as outlined in items 1 and 2 listed above (unilateral findings in children are sufficient for a definitive diagnosis, whereas unilateral findings in adults indicate a diagnosis of probable MMD); and 4) exclusion of known diseases with similar angiographic findings (for example, atherosclerosis, autoimmune disease, meningitis, brain tumors, Down's syndrome, neurofibromatosis Type 1, head injury, and cerebrovascular lesions after head irradiation). ${ }^{23}$

Information on patient sex, age at enrollment, age at onset of symptoms, age at diagnosis, family history of MMD, initial symptomatic and radiographic presentations at diagnosis, and neurological complications was obtained by ret- rospective medical record review or by interview with the patients or parents. Initial symptomatic presentation at diagnosis was defined by the most severe neurological symptom, and these symptoms were classified into intracranial hemorrhage/intraventricular hemorrhage, cerebral infarction, transient ischemic attack (TIA), seizures, and headache. A pediatric neuroradiologist (H.W.G.) who had no prior knowledge of each patient's RNF213 genotype evaluated the preoperative angiographic stage of each hemisphere. This stage was determined based on the stage of the more severely affected hemisphere per the Suzuki classification. ${ }^{27}$ In addition to the Suzuki stage, bilateral vasculopathy and posterior cerebral artery (PCA) involvement, which are factors related to disease severity, were also assessed. Headache, TIA, motor weakness, epilepsy, and cognitive impairment were regarded as neurological complications in patients followed up for more than 1 year after diagnosis or after the previous revascularization operation.

For comparison, we used a historical Korean control group (294 subjects, 80 males and 214 females, with a mean age of $40.9 \pm 10.9$ years) from a previous study ${ }^{17}$ of large-scale screening for the c.14429G>A (p.R4810K) variant in the general Asian population. These controls did not have histories of stroke and included 46 participants who were screened by angiography.

The institutional review board of the Asan Medical Center in Seoul, Korea, approved this study. All subjects gave written informed consent, and for those considered too young to consent, their parents gave informed consent.

\section{Mutation Analysis of RNF213}

Genomic DNA was extracted from peripheral blood using a Puregene blood kit (Qiagen). The major RNF213 SNPs associated with East Asian incidences of MMD ${ }^{18}$ were assessed: c.12037G $>$ A (p.D4013N), c.14429G $>A$ (p.R4810K), c.14506G >A (p.D4836N), and c.14576G >A (p.R4859K). Exons 44, 60, and 62 and the exon-intron boundaries of $R N F 213$ were amplified by polymerase chain reaction and directly sequenced using an $\mathrm{ABI} 3130 \mathrm{xl}$ genetic analyzer (Applied Biosystems) according to the manufacturer's instructions. Sequences were compared with established human RNF213 sequences (GenBank Accession No. NM_001256071.1). The investigators involved in genotyping had no prior knowledge of the phenotypic data.

\section{Statistical Analyses}

The strength of the association between a variant of RNF213 (c.14429G>A, p.R4810K) and the risk of MMD was estimated using a historical control group (294 controls) and odds ratios with corresponding 95\% confidence intervals. ${ }^{17}$ Differences between normally distributed categorical or continuous variables with respect to the RNF213 genotype were assessed using chi-square tests (chi-square with Fisher's exact test when warranted) and unpaired Student t-tests, respectively. Nonnormally distributed data were assessed using Mann-Whitney U-tests. The Cox regression and logistic regression models were used to estimate the impact of RNF213 variants on clinical manifestations. Statistical analyses were conducted using 
SPSS software (version 18.0, SPSS Inc.). The significance level was set as 0.05 .

\section{Results \\ Identification of RNF213 Variants in Korean Patients With MMD}

RNF213 variants were detected in the 3 exons under investigation in $76.4 \%$ (126) of the 165 MMD patients. With the exception of 1 patient with 1 novel variant (heterozygous c.12086A>G, p.Q4029R), all patients had the c.14429G >A (p.R4810K) variant. Thirteen patients were homozygous (A/A) and 112 were heterozygous (G/A).

\section{Association of the R4810K Variant With the Risk of MMD in Korean Patients}

The genotype and allele frequencies of the c.14429G $>A$ (R4810K) variant in MMD patients and historical controls are shown in Table 1. The minor allele frequency of the c.14429G $>$ A variant was significantly higher in the MMD patient group (41.8\% [138/330]) than in the historical control population (1.36\% [8/588]; $\mathrm{p}<0.001)$. The c.14429G $>$ A (p.R4810K) variant substantially increased the risk for MMD, with an OR of 52.11 (95\% CI 25.08108.26, $\mathrm{p}<0.001)$. The minor allele also occurred significantly more frequently in childhood-onset patients (age at onset $<15$ years) than in the adult-onset group $(44.3 \%$ [117/264] vs 31.8\% [21/66]; $p=0.018)$. Variant c. $14429 G>A$ (p.R4810K) genotypes occurred more frequently in patients with a family history of MMD than in patients with no family history of the disease ( $p=0.001$; Fig. 1$)$. In addition, the minor allele frequency was higher in the group with a family history of MMD than in the group without (48.9\% [45/92] vs 39.1\% [93/238]; $p=0.037)$.

\section{Comparison of Clinical Phenotypes With Genotype}

The clinical characteristics of the $165 \mathrm{MMD}$ patients with wild-type $(\mathrm{G} / \mathrm{G})$, c.14429G $>$ A heterozygous $(\mathrm{G} / \mathrm{A})$, and c.14429G $>$ A homozygous (A/A) genotypes are shown in Table 2. The mean age at disease onset was significantly lower in the A/A group $(2.7 \pm 2.1$ years, range $0.8-6.9$ years) than in the G/A group $(12.1 \pm 11.8$ years, range 0.9 -
56.9 years; $p<0.001)$ or the $\mathrm{G} / \mathrm{G}$ group $(16.7 \pm 17.7$ years, range 0.6-65.2 years; $p=0.005$; Fig. 2). All 13 homozygous patients manifested MMD during childhood, and homozygosity was significantly associated with the very early onset of MMD (age at onset $<5$ years; $p<0.001$ ). Univariate Cox regression analysis also demonstrated a strong association between the homozygous variant and the early onset of MMD (HR 10.52, 95\% CI 5.24-21.12, p < 0.001). Cerebral infarction was more frequent in the $\mathrm{A} / \mathrm{A}$ group than in the $\mathrm{G} / \mathrm{A}$ or $\mathrm{G} / \mathrm{G}$ groups $(\mathrm{p}=0.004$ and $\mathrm{p}<0.001$, respectively), whereas TIA was more common in G/A and $\mathrm{G} / \mathrm{G}$ patients than in those with $\mathrm{A} / \mathrm{A}(\mathrm{p}=0.032$ and 0.048 , respectively; Fig. 1). Cognitive impairment was more frequent in the A/A group than in the $\mathrm{G} / \mathrm{A}$ or $\mathrm{G} / \mathrm{G}$ groups (46.2\% vs $6.3 \%$ or $12.5 \%$, respectively; $p<0.001$ and $p<$ 0.011 , respectively). Moyamoya disease patients with wildtype alleles were more likely to be neurologically asymptomatic than patients who carried the $c .14429 \mathrm{G}>\mathrm{A}$ variant ( $\mathrm{p}$ $=0.034$ ). Patients carrying the minor allele were also more likely to experience early-onset MMD and cerebral infarction at presentation than patients homozygous for the wildtype allele (early-onset MMD 53.5\% vs late-onset MMD $37.7 \%, \mathrm{p}=0.001$; cerebral infarction $49.3 \%$ vs no cerebral infarction $36.2 \%, \mathrm{p}=0.002$ ).

\section{Illustrative Cases}

Familial cases of MMD having homozygous and heterozygous c. $14429 \mathrm{G}>\mathrm{A}$ (p.R4810K) variants in $R N F 213$ showed a different clinical course and disease severity according to their genotypes (Fig. 3). The father (Case 1) with the heterozygous (G/A) genotype had late-onset (37 years old) MMD with a mild clinical course despite severe angiographic findings. In contrast, the index case (Case 2 ) with the homozygous (A/A) variant presented with an earlier onset (20 months of age) and rapid progressive disease, which resulted in significant neurological deficits, epilepsy, and cognitive impairment. The 7-year-old younger sister (Case 3) with the heterozygous variant had experienced her first TIA at the age of 3 years but has not suffered any infarction until now. Their mother has no clinical symptoms of vasculopathy, and her genotype was not investigated.

TABLE 1. Genotype and allele distribution of c.14429G>A (p.R4810K) of RNF213 in patients with MMD and historical controls

\begin{tabular}{|c|c|c|c|c|c|c|c|c|c|}
\hline \multirow[b]{2}{*}{ Group } & \multirow{2}{*}{$\begin{array}{c}\text { No. of } \\
\text { Subjects }\end{array}$} & \multicolumn{3}{|c|}{$\begin{array}{l}\text { Genotype } \\
\text { Frequency }\end{array}$} & \multirow{2}{*}{$\begin{array}{c}p \\
\text { Value }^{*}\end{array}$} & \multicolumn{2}{|c|}{ Allele Frequency (no. [\%]) } & \multirow{2}{*}{$\begin{array}{c}p \\
\text { Value† }\end{array}$} & \multirow[b]{2}{*}{ OR $(95 \% \mathrm{Cl})$} \\
\hline & & $\mathrm{G} / \mathrm{G}$ & $\mathrm{G} / \mathrm{A}$ & $\mathrm{A} / \mathrm{A}$ & & Minor, A & Major, G & & \\
\hline All MMD cases in this study & 165 & 40 & 112 & 13 & $<0.001$ & $138(41.8)$ & $192(58.2)$ & $<0.001$ & $52.11(25.08-108.26)$ \\
\hline Historical controls ${ }^{17}$ & 294 & 286 & 8 & 0 & & $8(1.4)$ & $580(98.6)$ & & \\
\hline Childhood-onset MMD & 132 & 28 & 91 & 13 & 0.018 & $117(44.3)$ & $147(55.7)$ & 0.018 & - \\
\hline Adult-onset MMD & 33 & 12 & 21 & 0 & & $21(31.8)$ & $45(68.2)$ & & \\
\hline Family history of MMD & & & & & 0.001 & & & 0.037 & - \\
\hline Yes & 46 & 3 & 41 & 2 & & $45(48.9)$ & $47(51.1)$ & & \\
\hline No & 119 & 37 & 71 & 11 & & $93(39.1)$ & $145(60.9)$ & & \\
\hline
\end{tabular}

$\mathrm{A} / \mathrm{A}=$ homozygous for the $\mathrm{c} .14429 \mathrm{G}>\mathrm{A}$ variant; $\mathrm{G} / \mathrm{A}=$ heterozygous for the $\mathrm{c} .14429 \mathrm{G}>\mathrm{A}$ variant; $\mathrm{G} / \mathrm{G}=$ homozygous for the wild-type allele.

${ }^{*} p<0.05$ using the $X^{2}$ test for the distribution of genotype.

$\dagger p<0.05$ using the t-test for allele frequency. 


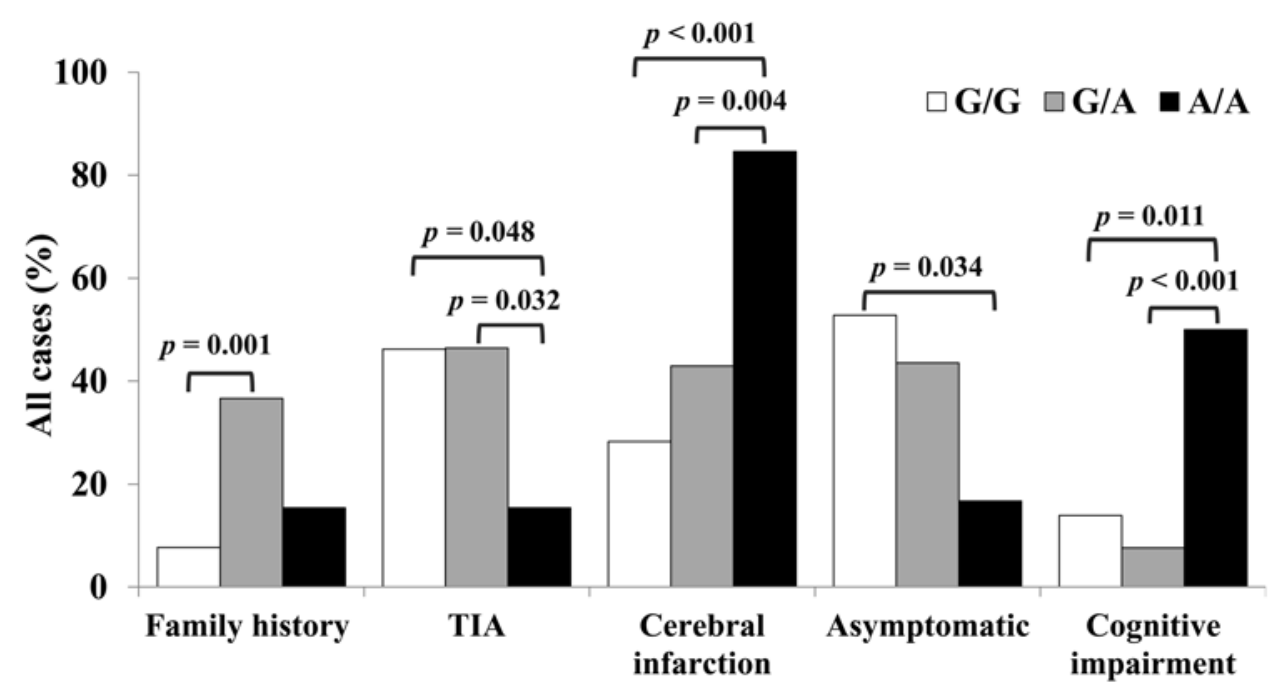

FIG. 1. Correlation between the c.14429G>A (p.R4810K) genotype of RNF213 and clinical phenotype. Family history of MMD, initial presentation at diagnosis, and neurological complications were compared among 3 patient groups with differing c.14429G $>A$ statuses: homozygous mutant $(A / A)$, heterozygous $(G / A)$, and homozygous wild type $(G / G)$. Significant results are shown.

\section{Discussion}

$R N F 213$ was previously identified as a susceptibility gene for MMD in East Asian populations. ${ }^{7,17,18,21,28}$ This study is the first to evaluate an association between the $R N F 213$ polymorphism and the phenotype of MMD in Korean patients. With the exception of a novel variant in 1 patient, the only variant identified in our cohort of 165 patients was c.14429G>A (p.R4810K). None of the patients harbored other major variants previously reported in East Asian populations..$^{18}$ The absence of the $\mathrm{c} .14576 \mathrm{G}>\mathrm{A}$ (p.R4859K) variant was particularly noted as it has been found in a high proportion of Japanese patients with MMD. ${ }^{21}$ This demonstrates the genetic variation underlying MMD susceptibility in different ethnic groups and

TABLE 2. Association of clinical characteristics with the c.14429G>A (p.R4810K) genotype of RNF213 in 165 patients with MMD

\begin{tabular}{|c|c|c|c|c|c|c|c|c|c|c|}
\hline \multirow[b]{3}{*}{ Factor } & \multirow[b]{3}{*}{$\begin{array}{c}\text { No. of } \\
\text { Subjects }\end{array}$} & \multicolumn{6}{|c|}{ Genotype } & \multirow{2}{*}{\multicolumn{2}{|c|}{ Allele Frequency (no. [\%]) }} & \multirow[b]{3}{*}{$\underset{\text { Value }}{p}$} \\
\hline & & \multirow[b]{2}{*}{$\begin{array}{c}G / G \\
(n=40)\end{array}$} & \multirow[b]{2}{*}{$\begin{array}{c}\mathrm{G} / \mathrm{A} \\
(\mathrm{n}=112)\end{array}$} & \multirow[b]{2}{*}{$\begin{array}{c}\text { A/A } \\
(n=13)\end{array}$} & \multicolumn{3}{|c|}{ p Value* } & & & \\
\hline & & & & & $\begin{array}{c}G / G \text { vs } \\
G / A\end{array}$ & $\begin{array}{c}G / G \text { vs } \\
A / A\end{array}$ & $\begin{array}{c}\text { G/A vs } \\
A / A\end{array}$ & Minor, A & Major, G & \\
\hline Female & 101 & 26 & 65 & 10 & NS & NS & NS & $85(42.1)$ & $117(57.9)$ & NS \\
\hline Age at onset $<5 \mathrm{yrs}$ & 43 & 8 & 24 & 11 & NS & $<0.001$ & $<0.001$ & $46(53.5)$ & $40(46.5)$ & 0.001 \\
\hline \multicolumn{11}{|c|}{ Initial presentations at diagnosis } \\
\hline TIA & 72 & 18 & 52 & 2 & NS & 0.048 & 0.032 & $56(38.9)$ & $88(61.1)$ & NS \\
\hline Cerebral infarction & 71 & 12 & 48 & 11 & NS & $<0.001$ & 0.004 & $70(49.3)$ & $72(50.7)$ & 0.002 \\
\hline $\mathrm{ICH} / \mathrm{IVH}$ & 8 & 4 & 4 & 0 & NS & NS & NS & $4(25.0)$ & $12(75.0)$ & NS \\
\hline Headache & 8 & 3 & 5 & 0 & NS & NS & NS & $5(31.2)$ & $11(68.8)$ & NS \\
\hline Seizures & 2 & 1 & 1 & 0 & NS & NS & NS & $1(25.0)$ & $3(75.0)$ & NS \\
\hline \multicolumn{11}{|l|}{ Angiographic findings } \\
\hline Mean Suzuki stage§ & 165 & $3.3 \pm 0.9$ & $3.2 \pm 0.9$ & $3.3 \pm 0.9$ & NS & NS & NS & & & \\
\hline Bilateral vasculopathy & 147 & 37 & 98 & 12 & NS & NS & NS & $122(41.5)$ & $172(58.5)$ & NS \\
\hline With PCA involvement & 37 & 9 & 24 & 4 & NS & NS & NS & $32(43.2)$ & $42(56.8)$ & NS \\
\hline \multicolumn{11}{|l|}{ Neurological complications } \\
\hline Asymptomatic & 61 & 19 & 40 & 2 & NS & 0.034 & NS & $44(36.1)$ & $78(63.9)$ & NS \\
\hline Headache & 49 & 13 & 32 & 4 & NS & NS & NS & $40(40.8)$ & $58(59.2)$ & NS \\
\hline TIA & 45 & 13 & 29 & 3 & NS & NS & NS & $35(38.9)$ & $55(61.1)$ & NS \\
\hline Cognitive impairment & 18 & 5 & 7 & 6 & NS & 0.011 & $<0.001$ & $19(52.8)$ & $17(47.2)$ & NS \\
\hline Motor weakness & 11 & 3 & 6 & 2 & NS & NS & NS & $10(45.4 \%)$ & $12(54.5 \%)$ & NS \\
\hline
\end{tabular}

$\mathrm{ICH} / \mathrm{IVH}=$ intracranial hemorrhage/intraventricular hemorrhage; $\mathrm{n}=$ total number of patients; $\mathrm{NS}=$ not significant.

${ }^{*} p<0.05$ using the $x^{2}$ test and t-test for clinical characteristics among 3 genotypes.

$\dagger p<0.05$ using the t-test for allele frequency between patients with and without each clinical characteristic.

$\S$ Mean values expressed with the standard deviation. 


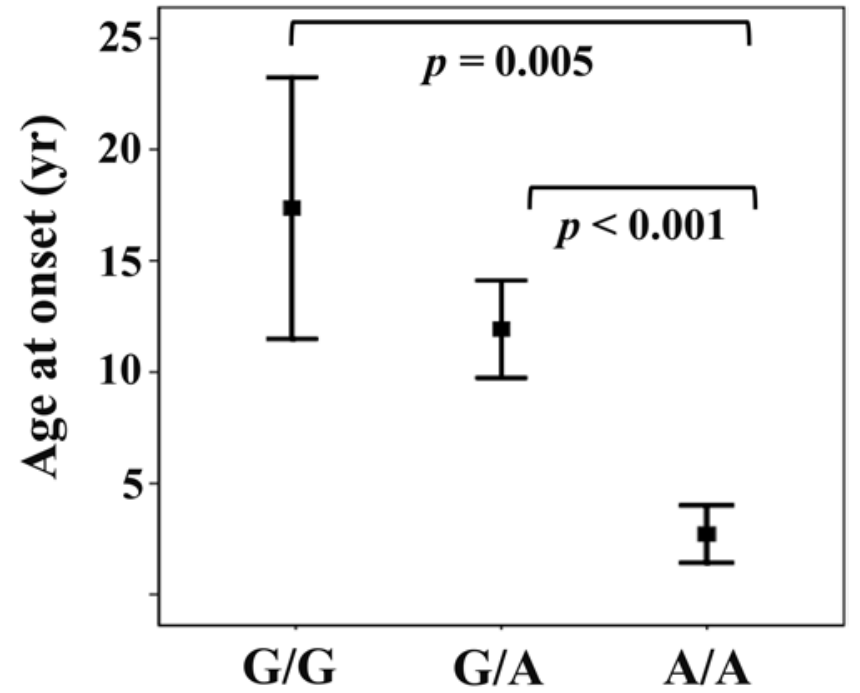

FIG. 2. Comparison of the mean age at onset among 3 groups of patients with differing c.14429G>A statuses of RNF213: homozygous mutant $(A / A)$, heterozygous $(G / A)$, and homozygous wild type $(G / G)$. Two groups were compared using unpaired Student t-tests.

also highlights the possible existence of other susceptibility variants in $R N F 213$. In comparing the allele frequency in our patient group with that in a historical control group, ${ }^{17}$ we found a significantly higher frequency of the minor allele (c.14429G >A, p.R4810K) in the Korean MMD group than in the control group ( $41.8 \%$ vs $1.4 \%)$. This frequency is comparable to those in a previous study in which $48.1 \%$ of Japanese MMD patients and 39.5\% of Korean MMD patients had this allele and in which the minor allele frequency of controls was $0.01 .{ }^{18}$ In the present study, patients with a family history of MMD were more likely to carry the c.14429G $>$ A allele than those with no family history. This finding supported previous research ${ }^{18,28}$ that showed c.14429G $>$ A (p.R4810K) to be one of the main genetic mutations associated with familial MMD.

In this study, the high prevalence of this allele in Korean patients with MMD was confirmed as well, and the clinical significance of the minor allele was also verified. The pathophysiological role of $R N F 213$ and the mechanisms by which $R N F 213$ polymorphisms lead to MMD have not been elucidated.Zebrafish lacking $R N F 213$ showed severely abnormal sprouting vessels in the head region, which suggested that RNF213 was involved in a novel signaling pathway during intracranial angiogenesis..$^{18} R N F 213$ deficiency in mice (RNF213-/-) was insufficient to induce MMD directly, despite the lack of functional RNF213. Nevertheless, vascular fragilities such as medial thinning were observed in the RNF213-/- mice, and these fragilities increased vulnerability to hemodynamic stress and secondary insults and thus facilitated the development of MMD. ${ }^{26}$ This finding provides some evidence for the role of RNF213 variants in the pathogenesis of MMD. However, genotype-phenotype correlation studies are necessary to further define the role of $R N F 213$ polymorphisms in the clinical setting of MMD. The minor allele occurs significantly more often in patients with childhood-onset MMD than in patients with adult-onset disease. Similar- ly, recent research by Miyatake and colleagues indicated that patients with the c.14576G >A (p.R4859K) variant had a significantly earlier disease onset than patients without it, and these patients were also more likely to experience more severe symptoms such as cerebral infarction and PCA stenosis. ${ }^{21}$ In addition, a study involving Chinese patients documented an association between the c.14429G $>A$ (p.R4810K) allele and the ischemic-type MMD and an association between g.115456G >A (p.A4399T) and the hemorrhagic-type MMD. ${ }^{28}$ These results corresponded with our findings that homozygous c.14429G $>A$ (p.R4810K) was significantly associated with a young age at disease onset, cerebral infarction at presentation, and poor cognitive outcomes.

Previous research has demonstrated that patients with a young age at disease onset have a more severe clinical course of MMD. ${ }^{8-10}$ In these patients, irreversible cerebral infarction at diagnosis was the most severe prognostic factor, underlining the importance of early diagnosis and surgical intervention. Our results revealed that unfavorable clinical prognostic factors, including early disease onset and cerebral infarction at diagnosis, were strongly associated with c.14429G $>$ A (p.R4810K) homozygosity. This finding suggests that homozygous c.14429G $>$ A (p.R4810K) may be of use as a specific biomarker for severe MMD with an unfavorable prognosis that requires early intervention for revascularization surgery. Thus diagnostic screening for the c.14429G $>$ A (p.R4810K) variant should be pursued in patients with MMD. Homozygous c.14429G $>$ A (p.R4810K) status was not associated with other putative poor prognostic factors in Japanese patients such as sex, angiographic findings of bilateral vasculopathy, or PCA involvement. ${ }^{8,14,22,29}$ Previous research in Korean children with MMD showed that unfavorable surgical outcomes were not associated with severe radiographic disease manifestations ${ }^{30}$ or PCA involvement. ${ }^{9}$ As noted above, the Korean and Japanese populations have similar but very different genetic backgrounds, particularly in RNF213 variants, which with other susceptibility genes may explain these discordant clinical findings. Then the genetic biomarkers can be used as a strong predictor of outcomes as well as the current radiological and hemodynamic markers for surgical intervention.

This study was limited by its retrospective design and the inclusion of only a relatively small number of adultonset cases. A further limitation was the examination of specific RNF213 exons rather than the whole gene. Only 1 patient with MMD and typical angiographic findings carried a variant other than c.14429G $>$ A (p.R4810K). Because RNF213 variants were not detected in 40 of the 165 MMD patients in this study, it is conceivable that these patients had distinct polymorphisms in other parts of the gene. Therefore, comprehensive genetic analysis of $R N F 213$ is necessary to determine whether other RNF213 variants are significant in the development of MMD.

\section{Conclusions}

Moyamoya disease is a significant cause of cerebral stroke in children and adults, especially in East Asia. This study indicates that the c.14429G $>$ A (p.R4810K) allele 
$\mathbf{A}$

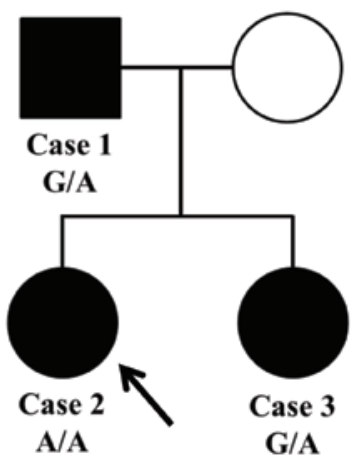

B

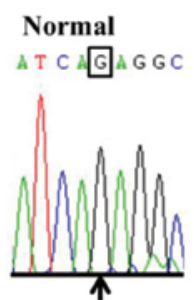

Case 2

A T A $A$ G G C

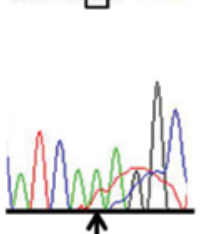

Case 1

A T C R G G

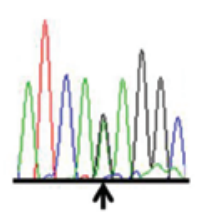

Case 3

A T C A R G G

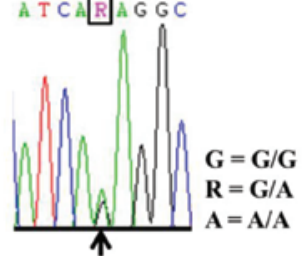

C. Case 1

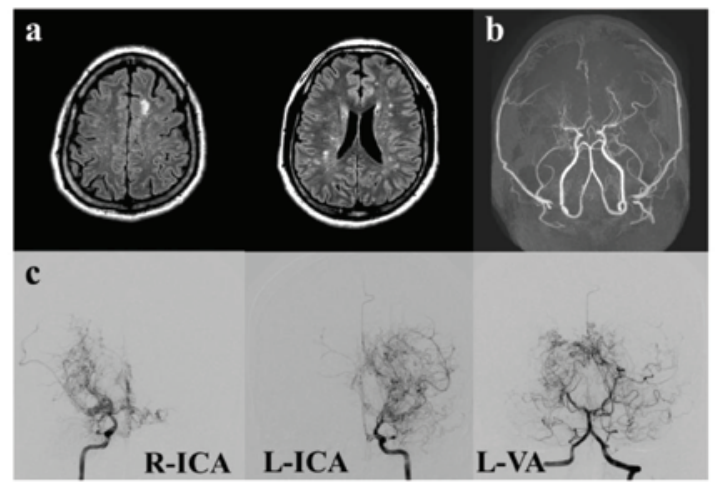

D. Case 2
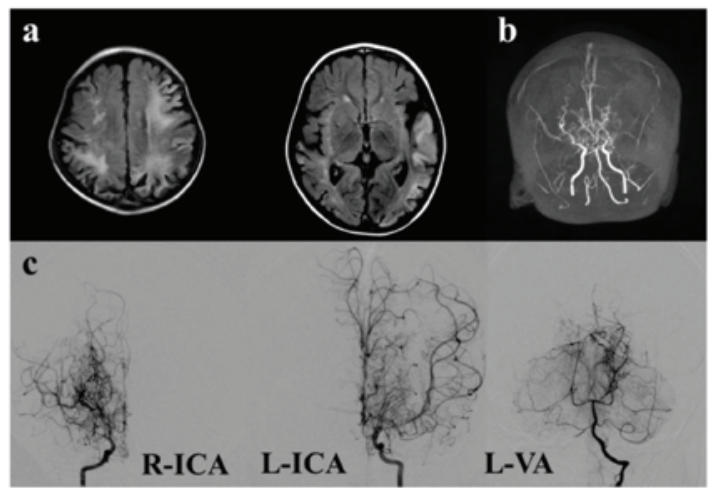

\section{E. Case 3}
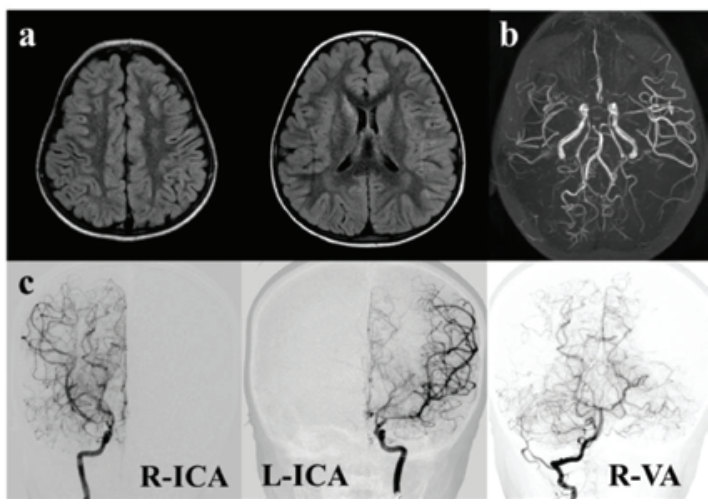

FIG. 3. Family pedigree (A): The index case (Case 2) has the homozygous c.14429G>A (p.R4810K) variant and the father (Case 1) and younger sister (Case 3 ) have the heterozygous $(\mathrm{G} / \mathrm{A})$ variant. Electropherograms $(\mathrm{B})$ of $R N F 213$ polymorphism showing the heterozygous variant $(\mathrm{G} / \mathrm{A})$ of Cases 1 and 3 and the homozygous variant $(\mathrm{A} / \mathrm{A})$ of Case 2 . Brain MR images and conventional angiographic findings at diagnosis (C-E). Case 1. High signal intensity lesion of the left centrum semiovale on FLAIR images (Ca) and tapered narrowing of the bilateral distal ICA, MCA, ACA, and PCA with multiple moyamoya vessels on MR angiography (MRA; $\mathrm{Cb}$ ) and cerebral angiography (Cc) in a 37-year-old man. Case 2. In a 20-month-old girl, FLAIR images (Da) showed widespread multifocal infarction of both cerebral hemispheres and MRA (Db) and cerebral angiography (Dc) demonstrated severe stenosis of the bilateral distal ICA, MCA, and ACA with extensive collateral formation. Case 3 . At the age of 3 years, this female patient showed no definite lesion of infarction on FLAIR images (Ea) and severe stenosis of the bilateral distal ICA, MCA, and ACA on MRA (Eb) and cerebral angiography $(E c) . L=$ left; $R=$ right; $V A=$ vertebral artery. Figure is available in color online only.

is strongly associated with Korean patients with MMD. The homozygous c.14429G $>$ A (p.R4810K) variant is particularly related to early-onset MMD, severe symptomatic manifestations at diagnosis, and poor prognosis. Therefore, the c.14429G >A (p.R4810K) homozygous variant can be considered as a biomarker for early surgical interven- tion in Korean MMD patients to prevent devastating neurological complications.

\section{References}

1. Bigi S, Fischer U, Wehrli E, Mattle HP, Boltshauser E, Bürki 
S, et al: Acute ischemic stroke in children versus young adults. Ann Neurol 70:245-254, 2011

2. Guo DC, Papke CL, Tran-Fadulu V, Regalado ES, Avidan N, Johnson RJ, et al: Mutations in smooth muscle alpha-actin (ACTA2) cause coronary artery disease, stroke, and Moyamoya disease, along with thoracic aortic disease. Am J Hum Genet 84:617-627, 2009

3. Hitomi T, Habu T, Kobayashi H, Okuda H, Harada KH, Osafune K, et al: Downregulation of Securin by the variant RNF213 R4810K (rs112735431, G>A) reduces angiogenic activity of induced pluripotent stem cell-derived vascular endothelial cells from moyamoya patients. Biochem Biophys Res Commun 438:13-19, 2013

4. Ikeda H, Sasaki T, Yoshimoto T, Fukui M, Arinami T: Mapping of a familial moyamoya disease gene to chromosome 3p24.2-p26. Am J Hum Genet 64:533-537, 1999

5. Im SH, Cho CB, Joo WI, Chough CK, Park HK, Lee KJ, et al: Prevalence and epidemiological features of moyamoya disease in Korea. J Cerebrovasc Endovasc Neurosurg 14:75-78, 2012 [Erratum in J Cerebrovasc Endovasc Neurosurg 14:262, 2012]

6. Inoue TK, Ikezaki K, Sasazuki T, Matsushima T, Fukui M: Linkage analysis of moyamoya disease on chromosome 6 . J Child Neurol 15:179-182, 2000

7. Kamada F, Aoki Y, Narisawa A, Abe Y, Komatsuzaki S, Kikuchi A, et al: A genome-wide association study identifies RNF213 as the first Moyamoya disease gene. J Hum Genet 56:34-40, 2011

8. Karasawa J, Touho H, Ohnishi H, Miyamoto S, Kikuchi H: Long-term follow-up study after extracranial-intracranial bypass surgery for anterior circulation ischemia in childhood moyamoya disease. J Neurosurg 77:84-89, 1992

9. Kim SK, Cho BK, Phi JH, Lee JY, Chae JH, Kim KJ, et al: Pediatric moyamoya disease: An analysis of 410 consecutive cases. Ann Neurol 68:92-101, 2010

10. Kim SK, Seol HJ, Cho BK, Hwang YS, Lee DS, Wang KC: Moyamoya disease among young patients: its aggressive clinical course and the role of active surgical treatment. Neurosurgery 54:840-846, 2004

11. Kim SK, Wang KC, Kim DG, Paek SH, Chung HT, Han $\mathrm{MH}$, et al: Clinical feature and outcome of pediatric cerebrovascular disease: a neurosurgical series. Childs Nerv Syst 16:421-428, 2000

12. Kuriyama S, Kusaka Y, Fujimura M, Wakai K, Tamakoshi A, Hashimoto S, et al: Prevalence and clinicoepidemiological features of moyamoya disease in Japan: findings from a nationwide epidemiological survey. Stroke 39:42-47, 2008

13. Kuroda S, Houkin K: Moyamoya disease: current concepts and future perspectives. Lancet Neurol 7:1056-1066, 2008

14. Kurokawa T, Tomita S, Ueda K, Narazaki O, Hanai T, Hasuo $\mathrm{K}$, et al: Prognosis of occlusive disease of the circle of Willis (moyamoya disease) in children. Pediatr Neurol 1:274-277, 1985

15. Li H, Zhang ZS, Liu W, Yang WZ, Dong ZN, Ma MJ, et al: Association of a functional polymorphism in the MMP-3 gene with moyamoya disease in the Chinese Han population. Cerebrovasc Dis 30:618-625, 2010

16. Liu W, Hashikata H, Inoue K, Matsuura N, Mineharu Y, Kobayashi H, et al: A rare Asian founder polymorphism of Raptor may explain the high prevalence of moyamoya disease among East Asians and its low prevalence among Caucasians. Environ Health Prev Med 15:94-104, 2010

17. Liu W, Hitomi T, Kobayashi H, Harada KH, Koizumi A: Distribution of moyamoya disease susceptibility polymorphism p.R4810K in RNF213 in East and Southeast Asian populations. Neurol Med Chir (Tokyo) 52:299-303, 2012

18. Liu W, Morito D, Takashima S, Mineharu Y, Kobayashi H, Hitomi T, et al: Identification of RNF213 as a susceptibility gene for moyamoya disease and its possible role in vascular development. PLoS One 6:e22542, 2011

19. Mackay MT, Wiznitzer M, Benedict SL, Lee KJ, Deveber GA, Ganesan V: Arterial ischemic stroke risk factors: the International Pediatric Stroke Study. Ann Neurol 69:130-140, 2011

20. Mineharu Y, Takagi Y, Takahashi JC, Hashikata H, Liu W, Hitomi T, et al: Rapid progression of unilateral moyamoya disease in a patient with a family history and an RNF213 risk variant. Cerebrovasc Dis 36:155-157, 2013

21. Miyatake S, Miyake N, Touho H, Nishimura-Tadaki A, Kondo Y, Okada I, et al: Homozygous c.14576G $>$ A variant of RNF213 predicts early-onset and severe form of moyamoya disease. Neurology 78:803-810, 2012

22. Mugikura S, Higano S, Shirane R, Fujimura M, Shimanuki Y, Takahashi S: Posterior circulation and high prevalence of ischemic stroke among young pediatric patients with Moyamoya disease: evidence of angiography-based differences by age at diagnosis. AJNR Am J Neuroradiol 32:192-198, 2011

23. Research Committee on the Pathology and Treatment of Spontaneous Occlusion of the Circle of Willis: Guidelines for diagnosis and treatment of moyamoya disease (spontaneous occlusion of the circle of Willis). Neurol Med Chir (Tokyo) 52:245-266, 2012

24. Sakurai K, Horiuchi Y, Ikeda H, Ikezaki K, Yoshimoto T, Fukui M, et al: A novel susceptibility locus for moyamoya disease on chromosome 8q23. J Hum Genet 49:278-281, 2004

25. Scott RM, Smith ER: Moyamoya disease and moyamoya syndrome. N Engl J Med 360:1226-1237, 2009

26. Sonobe S, Fujimura M, Niizuma K, Nishijima Y, Ito A, Shimizu H, et al: Temporal profile of the vascular anatomy evaluated by 9.4-T magnetic resonance angiography and histopathological analysis in mice lacking RNF213: a susceptibility gene for moyamoya disease. Brain Res 1552:64-71, 2014

27. Suzuki J, Kodama N: Moyamoya disease-a review. Stroke 14:104-109, 1983

28. Wu Z, Jiang H, Zhang L, Xu X, Zhang X, Kang Z, et al: Molecular analysis of RNF213 gene for moyamoya disease in the Chinese Han population. PLoS One 7:e48179, 2012

29. Yamada I, Himeno Y, Suzuki S, Matsushima Y: Posterior circulation in moyamoya disease: angiographic study. Radiology 197:239-246, 1995

30. Yamauchi T, Houkin K, Tada M, Abe H: Familial occurrence of moyamoya disease. Clin Neurol Neurosurg 99 (Suppl 2):S162-S167, 1997

31. Yamauchi T, Tada M, Houkin K, Tanaka T, Nakamura Y, Kuroda $\mathrm{S}$, et al: Linkage of familial moyamoya disease (spontaneous occlusion of the circle of Willis) to chromosome $17 \mathrm{q} 25$. Stroke 31:930-935, 2000

\section{Disclosure}

The authors report no conflict of interest concerning the materials or methods used in this study or the findings specified in this paper.

\section{Author Contributions}

Conception and design: Ra, Yoo. Acquisition of data: EH Kim, Park, Ahn, Goo. Analysis and interpretation of data: EH Kim, Yum, GH Kim. Drafting the article: EH Kim, Yum, Yoo. Critically revising the article: Yum. Reviewed submitted version of manuscript: Ra, GH Kim, Ko. Statistical analysis: EH Kim. Study supervision: Ra, Ko, Yoo.

\section{Correspondence}

Young-Shin Ra, Department of Neurosurgery, Asan Medical Center, College of Medicine, University of Ulsan, 88 Olympicro 43-gil, Songpa-gu, Seoul 138-736, Republic of Korea. email: youngshin.ra@gmail.com. 